\title{
All-Weather Perception for Man-Portable Robots Using Ultra-Wideband Radar
}

\author{
Brian Yamauchi
}

\begin{abstract}
Autonomous man-portable robots have the potential to provide a wide range of new capabilities for both military and civilian applications. Previous research in autonomy for small robots has focused on vision, LIDAR, and sonar sensors. While vision and LIDAR work well in clear weather, they are seriously impaired by rain, snow, fog, and smoke. Sonar can penetrate adverse weather, but has limited range outdoors, and suffers from specular reflections indoors. For the Daredevil Project, we have investigated the use of ultrawideband (UWB) radar to provide obstacle detection capabilities for man-portable robots. Our research shows that UWB radar can effectively penetrate adverse weather, including dense fog, and detect obstacles that would be undetectable by vision or LIDAR under the same conditions. We have developed filtering algorithms that process the raw radar returns to eliminate reflections from ground clutter and make obstacles easier to detect. We have tested this system on an iRobot PackBot equipped with both UWB radar and LIDAR, and we have demonstrated how UWB radar can be used for obstacle detection in obscured environments.
\end{abstract}

\section{INTRODUCTION}

$\mathrm{S}_{\mathrm{r} e \mathrm{~s}}^{\mathrm{N}}$ MALL unmanned ground vehicles (UGVs) have revolutionized the way in which improvised explosive devices (IEDs) are disarmed by explosive ordnance disposal (EOD) technicians. Thousands of man-portable UGVs, such as the iRobot PackBot, have been deployed to Iraq and Afghanistan, where they have saved many lives. Other robots, such as the Future Combat Systems (FCS) Small Unmanned Ground Vehicle (SUGV) developed by iRobot, are bringing remote reconnaissance capabilities to the broader US Army infantry forces.

The small UGVs currently deployed on the battlefield are teleoperated by a remote operator who must control all of the robot's actions via a video link. This requires the operator's full attention and prevents the operator from conducting other tasks. Often, another soldier is required to protect the operator from any threats in the vicinity.

One of the next steps in battlefield robotics will be to enable robots to navigate autonomously, allowing the operator to direct the robot using high-level commands (e.g. "Navigate to location X.") and freeing the operator to

Manuscript received September 8, 2009. This work was supported in part by the U.S. Army Tank-automotive and Armaments Research, Development, and Engineering Center (TARDEC) under Grant W56HZV07-C-0519.

Brian Yamauchi is with iRobot Research, iRobot Corporation, 8 Crosby Drive, M/S 8-1, Bedford, MA 01730 USA (phone: 781-430-3291, fax: 781430-3898, email: yamauchi@irobot.com). conduct other tasks. Autonomous navigation is a key capability that is required to achieve force multiplication (i.e. allowing one operator to control many robots).

Much previous research has been conducted in mobile robot navigation, including some work with man-portable robots [1]-[4]. These robots typically use sensors such as vision, LIDAR, and sonar to perceive the world and avoid collisions. While vision and LIDAR work well in clear weather, they have serious limitations when dealing with rain and snow, and they are unable to see through thick smoke or fog. Sonar is able to operate in adverse weather and penetrate smoke and fog. However, sonar has limited range when used in the relatively sparse medium of air (as opposed to the dense medium of water). In addition, when a sonar pulse hits a flat surface, such as building wall, at a shallow angle, it often reflects away from the sensor (i.e. specular reflection), resulting in either an erroneously long range reading or no reading at all.

Radar offers the capability to detect obstacles through rain, snow, and fog without the limitations of sonar. Radarbased Adaptive Cruise Control (ACC) and active brake assist systems are available as factory options for luxury automobiles from Audi, BMW, Cadillac, Lexus, and Mercedes, among others [5]. ACC systems monitor the range to the vehicle ahead and adjust the throttle to maintain a constant following distance, while active brake assist systems provide additional braking force if a collision is imminent.

For the Daredevil Project [6], we are investigating the use of UWB radar to provide all-weather perception capabilities for the man-portable iRobot PackBot UGV. Unlike conventional radar, which transmits relatively long pulses of RF energy within a narrow frequency range, UWB radar sends an extremely short pulse of RF energy across a wide range of frequencies. The brief duration of each pulse results in improved range resolution, immunity to passive interference (e.g. rain, fog, aerosols), and the ability to detect targets that are stationary with respect to the sensor [7].

\section{RELATED WORK}

In [6], we described our initial experiments with UWB radar. These experiments showed that UWB radar could reliably detect obstacles in a snowstorm, through dense fog, and through sparse foliage.

Other researchers have developed obstacle avoidance and navigation techniques for man-portable robots using vision, LIDAR, and sonar. Konolige developed sonar-based reactive 
navigation capabilities for the inexpensive ERRATIC robot that won second-place in the 1994 AAAI Robot Competition [1]. Researchers at the Jet Propulsion Laboratory (JPL), Carnegie Mellon University (CMU), iRobot, and the University of Southern California (USC) developed autonomous navigation capabilities for the Urban Robot (a predecessor to the iRobot PackBot) using vision and LIDAR [2]. As part of the Small Robot Technology Transfer Program, the US Navy Space and Naval Warfare Systems Command (SPAWAR) and the Idaho National Laboratory (INL) transitioned algorithms for obstacle avoidance, mapping, localization, and path planning to several different small robots, including the iRobot PackBot [3].

Automotive radars have been used as sensors for a number of autonomous vehicles, including several entrants in the DARPA Urban Challenge. The winning vehicle, CMU's Boss, used a Continental ARS 300 automotive radar to measure the velocity of other vehicles [8]. Stanford's Junior used five BOSCH Long Range Radars to detect moving objects in intersections [9]. MIT's Talos vehicle used fifteen Delphi ACC millimeter wave radars to detect fastapproaching vehicles at long range [10].

These automotive radars differ in several fundamental ways from the UWB radar used by Daredevil. Automotive radars are optimized for detecting obstacles at long range (up to $200 \mathrm{~m}$ ) with a typical range resolution of $1 \mathrm{~m}$ and a typical range accuracy of 5\% [11]. In general, these radars return multiple tracks for the strongest targets. However, as Leonard points out [10], they are unable to reliably detect the difference between small objects (e.g. a metal bolt, a sewer grate) and large objects (e.g. cars). For that reason, all of these teams used radar primarily to detect moving objects, since any object moving at high speeds was almost certainly another vehicle under the conditions of the Urban Challenge.

In contrast, the Multispectral Solutions (MSSI) Radar Developer's Kit Lite (RaDeKL) UWB radar used by Daredevil was designed for precise ranging at short to medium range, providing $0.3 \mathrm{~m}(1 \mathrm{ft})$ resolution at ranges up to $78 \mathrm{~m}(256 \mathrm{ft})$. Instead of providing processed radar tracks, the RaDeKL radar provides the raw radar strength measured in each $0.3 \mathrm{~m}$ long range bin. As a result, the radar return can be used to measure the size and shape of obstacles. In addition, the RaDeKL radar is suitable for use indoors as well as outdoors, which is a key advantage for man-portable robots that are often used for indoor applications.

\section{DAREDEVIL PACKBot}

Fig. 1 shows the Daredevil PackBot, which is built upon the rugged, man-portable, combat-proven iRobot PackBot platform. For Daredevil, we added an MSSI RaDeKL UWB radar sensor mounted on a TRACLabs Biclops pan/tilt mount, which in turn is mounted on a $1 \mathrm{~m}$ tall mast. The mast exists to raise the sensor and reduce the amount of energy reflected from ground clutter.

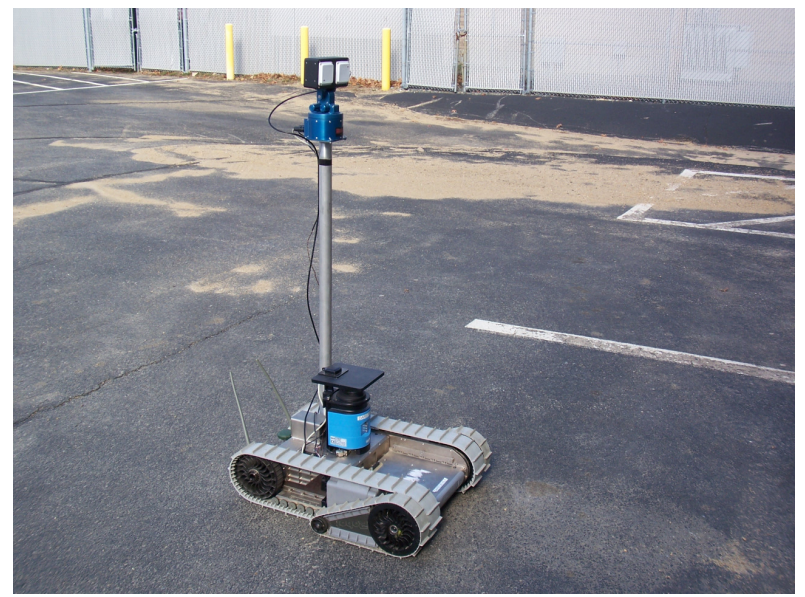

Fig. 1. Daredevil PackBot equipped with a RaDeKL UWB radar on a pan/tilt mount and a SICK LD OEM LIDAR.

The RaDeKL radar transmits an ultra wideband pulse (400 $\mathrm{MHz}$ wide) centered around $6.35 \mathrm{GHz}$, with an FCCapproved $30 \mathrm{~mW}$ peak power level. The brief duration of this pulse results in extremely low transmit power $(0.2 \mathrm{nW})$, which is equivalent to one ten-millionth of the power of a typical cell phone. The sensor requires a $1.2 \mathrm{~W}$ power input with a power voltage anywhere in the range of 7.2-35 V.

The sensor measures returned radar strength over sequential 2 ns time intervals, corresponding to round-trip

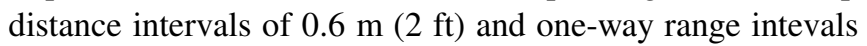

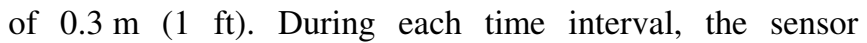
integrates the signal return strength and maps the resulting total to an 8-bit value (0-255). The sensor repeats this process 256 times, to measure the radar return strength at ranges from 0 to $78 \mathrm{~m}$ ( 0 to $255 \mathrm{ft})$. The sensor then publishes this 256-value array over its USB interface, which is based on FTDI drivers. An optional time delay can be specified before the sensor begins to register return signals, allowing the $78 \mathrm{~m}$ usable sensor distance to begin at a longer range from the sensor (up to $273 \mathrm{~m}$ ). However, at longer ranges, multipath from ground reflection becomes an increasing problem. In our experiments, we did not use any offset, and used the default minimum range of $0 \mathrm{~m}$ and maximum range of $78 \mathrm{~m}$.

The physical dimensions of the sensor are $15 \mathrm{~cm} \mathrm{x} 8 \mathrm{~cm} \mathrm{x}$ $6 \mathrm{~cm}(6 " \times 3.25 " \times 2.375 ")$. The radar has a field-of-view (FOV) that is $40^{\circ}$ degrees wide along the horizontal axis and $40^{\circ}$ degrees wide along the vertical axis. Both the transmit power and receiver sensitivity can be adjusted on-the-fly by commands over the USB interface.

We mounted the RaDeKL radar on a Biclops PT pan/tilt base manufactured by TRACLabs. The pan/tilt unit provides $360^{\circ}$ degree coverage along the pan axis $\left( \pm 180^{\circ}\right)$ and $180^{\circ}$ degree range of motion along the tilt axis $\left( \pm 90^{\circ}\right)$. The angular resolution of the pan/tilt encoders is $0.018^{\circ}$. The pan/tilt unit requires a $24 \mathrm{~V}$ power supply at $1 \mathrm{~A}$ and is controlled via a USB interface. Power for both the RaDeKL and the Biclops PT was provided by the PackBot's onboard power system. 


\section{UWB RADAR FILTER ALGORITHMS}

\section{A. Raw Radar Returns}

We developed a real-time viewer for the scanning RaDeKL UWB radar mounted on the Biclops pan/tilt mount. Fig. 2 shows an overhead view of a radar scan. In this image, brighter areas correspond to stronger returns. The radar is located at the center of the image, and the concentric circles are spaced at $1 \mathrm{~m}$ intervals. The bright line indicates the current bearing of the radar.

For these experiments, we rotated the radar $360^{\circ}$ (panning left and right) at a speed of 0.1 radians/second. Full power was used for the radar transmitter $(0 \mathrm{~dB})$, while the radar receiver was attenuated by $-20 \mathrm{~dB}$ to reduce noise.

Radar readings were received at an average rate of $10 \mathrm{~Hz}$, so the average angular separation between readings was roughly $0.5^{\circ}$. Each reading consisted of the return strength for the 256 range bins (each $0.3 \mathrm{~m}$ long) along the current bearing of the radar. For each bin, we drew a square area at the corresponding location, with the brightness of the area corresponding to strength of the radar return. Unlike a grid representation, the $(x, y)$ center of each region is not quantized, since the current sensor bearing is a continuous floating-point value.

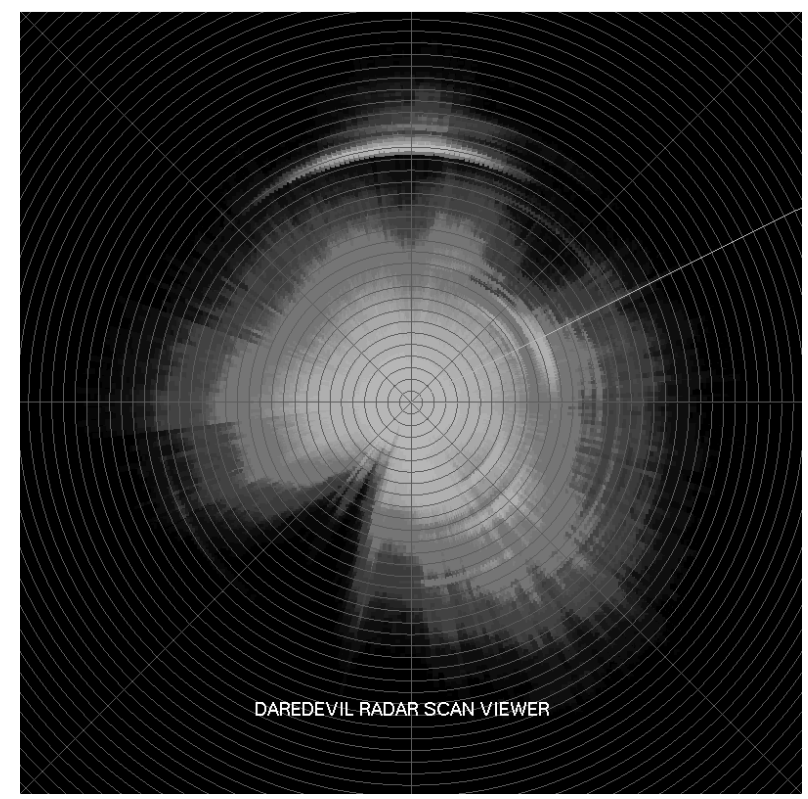

Fig. 2. Raw radar return strength from RaDeKL UWB radar positioned $1 \mathrm{~m}$ above pavement, with maximum transmit power and $-20 \mathrm{~dB}$ receiver sensitivity. Concentric circles are spaced at $1 \mathrm{~m}$ intervals centered on radar. Brighter areas represent stronger return signals. Bright area at center is the result of ground clutter. Bright arc at top center corresponds to a concrete wall.

Fig. 2 shows the difficulty of directly interpreting the raw radar returns. The wide area of strong returns near the sensor is due to reflections from ground clutter. The large, bright arc at the top of the image is a concrete wall. The bright area on the top right of the image is a shipping container. The laptop controlling the radar is just to the left and below the radar at the center of the image.
Our experiments showed that the radar can detect some obstacles reliably (e.g. walls), but that there is a large amount of energy being returned to the sensor from the ground clutter close to the sensor. These experiments were conducted in an open parking lot, with the sensor mounted one meter above the ground, oriented parallel to the ground, and horizontally polarized. Based on these experiments, we concluded that additional filtering was required to facilitate the interpretation of radar data.

\section{B. Delta Filter Algorithm (DFA)}

We have developed the delta filter algorithm (DFA) to reduce the effects of ground clutter and better identify true obstacles in the sensor data. The DFA works by examining the radar return bins in order from the sensor outward. If the sensor reading for the current bin exceeds the reading from the previously examined bin by greater than a threshold value $\delta$, the bin location is marked as occupied. Otherwise, the bin location is marked as empty.

If $\mathrm{raw}_{i}$ is the value of bin $i$, then the corresponding delta filter value is given by (1).

$$
\text { delta }_{i}=\left\{\begin{array}{cc}
1 & \text { if } \text { raw }_{i}-\text { raw }_{i-1}>\delta \\
0 & \text { otherwise }
\end{array}\right.
$$

We performed a set of experiments comparing UWB radar data using DFA to SICK LIDAR range data in an urban environment (parking lot). Our experiments show that by applying the DFA, we can obtain accurate range readings from the UWB radar that closely approximate the LIDAR data. In addition, our experiments show that the UWB radar is able to see through fences and detect the obstacles behind. These experiments were performed with the delta threshold set to $1(\delta=1)$, transmit attenuation set to $-5 \mathrm{~dB}$, and receiver sensitivity set to maximum $(0 \mathrm{~dB})$.

In the next experiment, we positioned the Daredevil PackBot approximately $15 \mathrm{~m}$ from a chain link fence with white plastic slats forming an opaque barrier. Fig. 5 shows the UWB radar data output by the DFA filter (green) superimposed on SICK LIDAR data (red) collected at the same time. Grid lines are spaced at $10 \mathrm{~m}$ intervals. (Note that the apparent stair-stepping is an artifact of the way this image was rendered, with overlapping squares for the radar points. The actual range data shows smooth arcs.)

At longer ranges, reflections from the wall are represented by arcs rather than lines. This is due to the large $40^{\circ}$ horizontal FOV of the sensor couple with the discretization of the range values. In the future, we plan to accumulate multiple sensor readings over time using an occupancy grid [12], which should eliminate this effect. Fig. 3 shows that the UWB radar using DFA can detect both the fence and the building wall behind. In comparison, the LIDAR detects only the fence and not the building. 


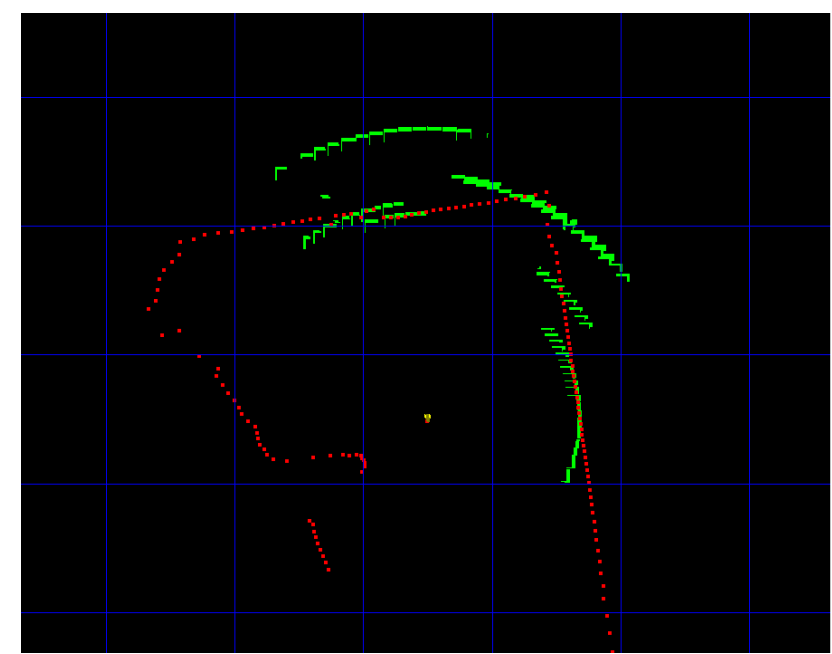

Fig. 3. DFA-filtered UWB radar (green) and LIDAR (red) data from chain link fence in front of building

\section{Max Filter Algorithm (MFA)}

The second filtering algorithm we developed was the $\max$ filter algorithm (MFA). The MFA examines all of the radar bins in a given return and returns a positive reading for the bin with the maximum return strength, if that bin is farther than a minimum range threshold. If the maximum return strength is for a bin that is closer than the minimum range threshold, the filter returns a null reading. If more than one reading has the maximum value, the MFA returns the closest reading, if the range to that reading is over the minimum range threshold, and the null reading otherwise.

The MFA is a very effective method for finding the strongest radar reflectors in an environment with many reflections. Fig. 4 shows the results from an indoor experiment using the MFA with the radar scanning $360^{\circ}$ from a fixed location at the center of the hallway intersection. The green points show the ranges returned by the MFA. The red points show the LIDAR returns. The blue lines are spaced at $10 \mathrm{~m}$ intervals.

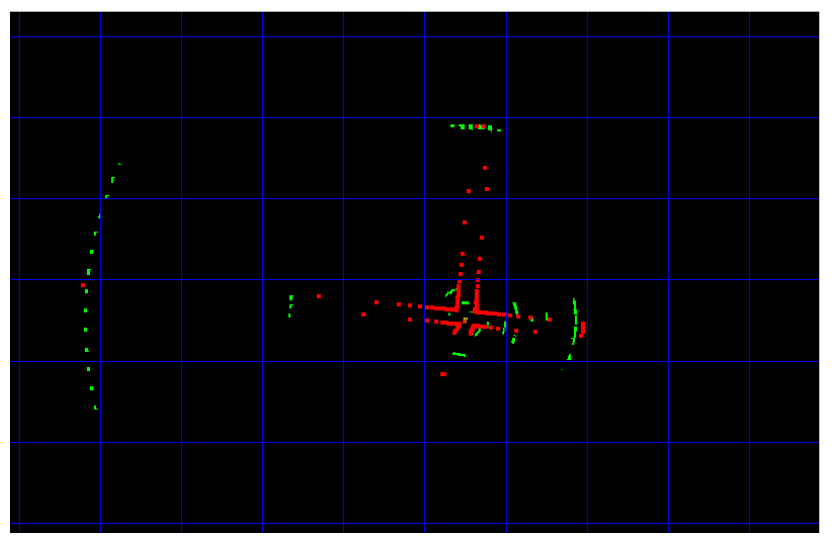

Fig. 4. MFA-filtered UWB radar detects doors at ranges up to $45 \mathrm{~m}$ (green $=$ radar, red $=$ LIDAR, blue lines space at $10 \mathrm{~m}$ intervals)

As shown by Fig. 4, we were able to detect closed doors at the ends of these hallways at ranges of up to 45 meters. Note that in the case of the left door, the LIDAR was only able to get a single return, while the UWB radar was able to return multiple returns. At the same time, this figure shows the relatively low angular resolution of the radar sensor. In future work, we plan to use techniques such as occupancy grids [12] to accumulate radar data over multiple returns and provide a more precise estimation of target location based on the probabilistic sensor models.

\section{Calibrated Max Filter Algorithm (CMFA)}

Our most recent and best performing filter algorithm is the calibrated max filter algorithm (CMFA), a modified version of the MFA. The purpose of the CMFA is to eliminate the ambient reflections from the ground plane, which are stronger close to the sensor and weaker farther from the sensor. In the initial MFA, the minimum detection range had to be set farther from the sensor to ignore the reflections from ground clutter, but this prevented the MFA from detecting close-range obstacles. The CMFA is able to detect closer objects by subtracting the ambient reflection (with no obstacle present). Any remaining signal above ambient indicates the presence of an obstacle.

In the calibration stage of the CMFA, the radar is first aimed at open space in the current environment. A series of raw radar readings is returned and the average value of each bin is stored in the calibration vector (2).

$$
c_{i}=\frac{1}{n} \sum_{j=1 . . n} r_{i j}
$$

In (2), $c_{i}$ is element $i$ of the calibration vector, $r_{i j}$ is bin $i$ from raw radar scan $j$, and $n$ is the number of raw scans stored. For our experiments, we averaged over 20 raw radar scans, which were acquired over 2-4 seconds.

During operation of the robot, the calibration vector is then subtracted from each raw range scan and the result is stored in an adjusted range vector (3).

$$
a_{i}=\left\{\begin{array}{cc}
0 & \text { if } r_{i}<c_{i} \\
r_{i}-c_{i} & \text { otherwise }
\end{array}\right.
$$

In (3), $a_{i}$ is element $i$ of the adjusted range vector, $r_{i}$ is bin $i$ of the raw range vector, and $c_{i}$ is element $i$ of the calibration vector.

The MFA is then applied to the adjusted range vector to determine to return the filtered range value. The index of the maximum element of the adjusted range vector is returned. If more than one element has the maximum value, the index of the bin closest to the sensor is returned.

In our experiments, we have found that the CMFA works significantly better than the MFA at detecting obstacles at close range. This is particularly useful for operations indoors and in cluttered environments.

\section{RADAR/LIDAR EXPERIMENTS IN FOG}

We conducted experiments to compare the capabilities of UWB radar and LIDAR in environments filled with waterbased fog. In these experiments, we determined that dense fog would completely obscure LIDAR and vision, but this fog had no effect on UWB radar returns. 


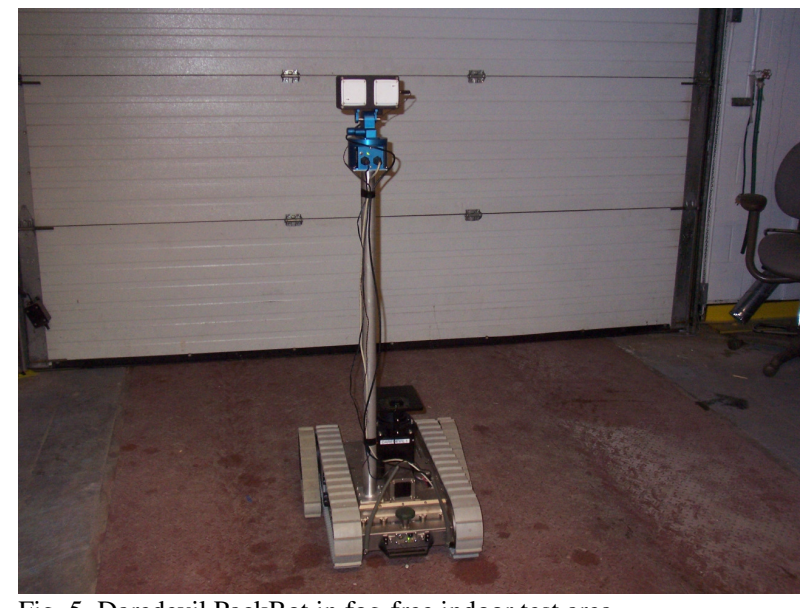

Fig. 5. Daredevil PackBot in fog-free indoor test area

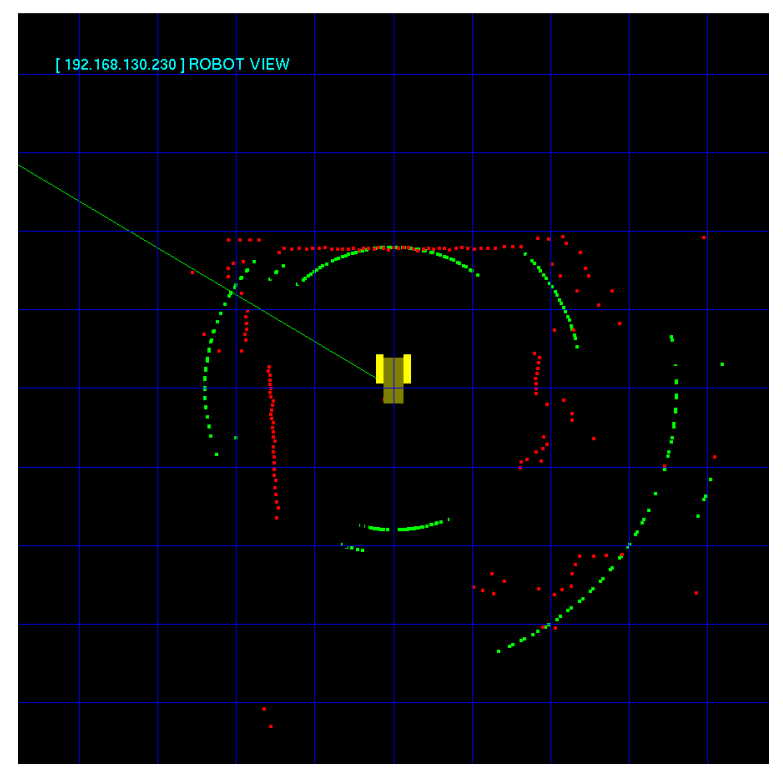

Fig. 6. CMFA-filtered UWB radar (green) and LIDAR (red) readings in clear air

The following figures show the Daredevil PackBot in our indoor test area. Initially the robot is in clear air. Then the fog machine is activated, and fog is allowed to fill the room until the robot is fully obscured and LIDAR and vision are completely blocked.

Fig. 5 shows the robot in the initial, fog-free environment. The radar rotates $360^{\circ}\left( \pm 180^{\circ}\right)$ alternating direction with each sweep. Fig. 6 shows the radar (green dots) and LIDAR (red dots) returns in this environment.

Both sensors are able to detect the obstacles in this environment, and the LIDAR shows considerably higher resolution and accuracy. In future work, we plan to increase the effective angular resolution of the radar using occupancy grid techniques [12], but LIDAR will always provide greater precision in clear air.

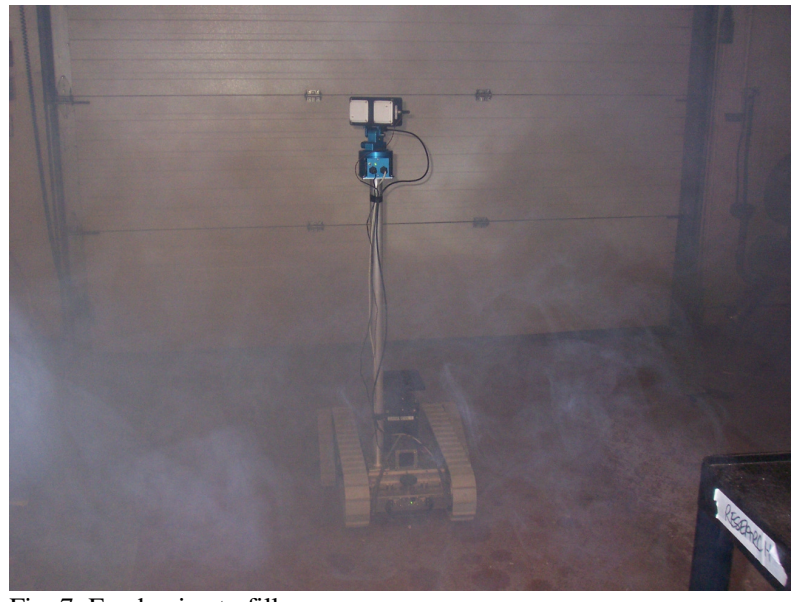

Fig. 7. Fog begins to fill area

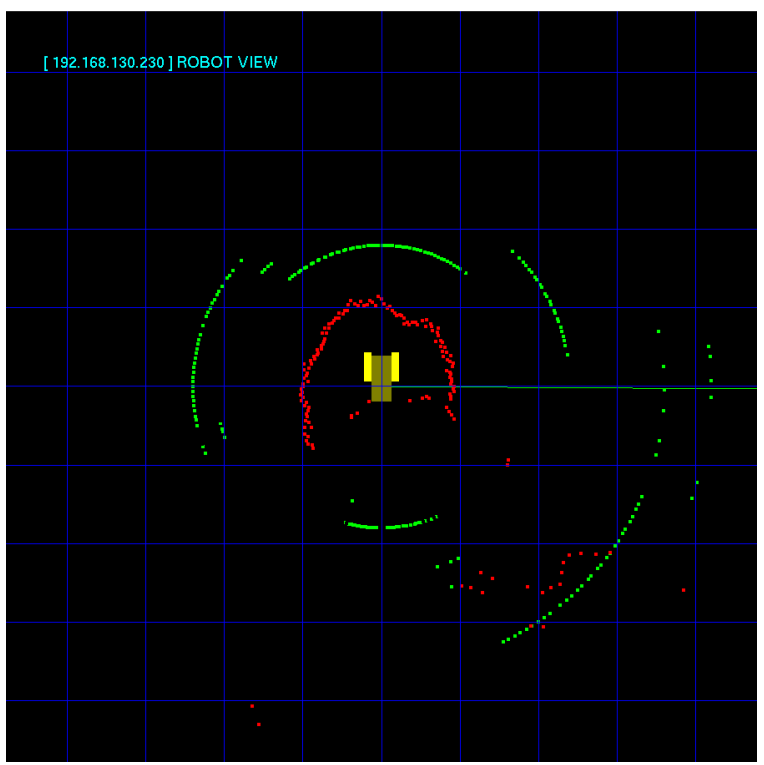

Fig. 8. CMFA-filtered UWB radar (green) and LIDAR (red) readings in partiallv fogged environment

Fig. 7 shows the test area after the fog machine is activated. Fog has begun to fill the area, and the robot is partially obscured. Fig. 8 shows the radar and LIDAR returns in this partially obscured environment. Even in this moderate fog density, LIDAR readings have already become almost useless.

In front and to the sides of the robot, the LIDAR can only penetrate this moderate fog to a depth of about $1 \mathrm{~m}$. Only behind the robot is the air sufficiently clear that the LIDAR can continue to detect some obstacles. Note that the radar returns in Fig. 8 are virtually identical to those in Fig. 6. This shows that the fog has not affected radar accuracy.

Fig. 9 shows the test area after it has been completely filled with dense fog. Optical sensors, including both LIDAR and vision, are completely useless in this environment. Fig. 9 shows the corresponding radar and LIDAR returns. The LIDAR can penetrate less than $1 \mathrm{~m}$ through the fog in all directions, and is incapable of detecting any obstacles beyond this range. At the same time, the radar readings in Fig. 10 are nearly identical to those in Fig. 6 (clear air). 


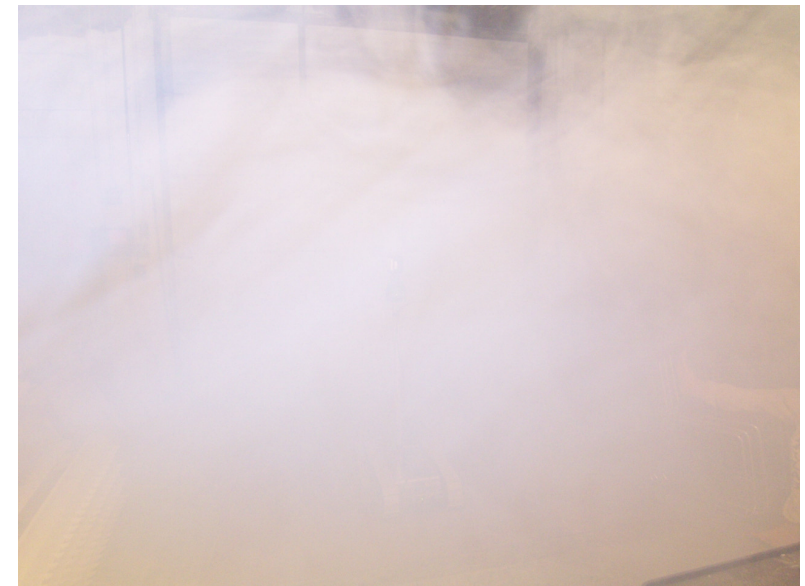

Fig. 9. Fog completely obscures robot

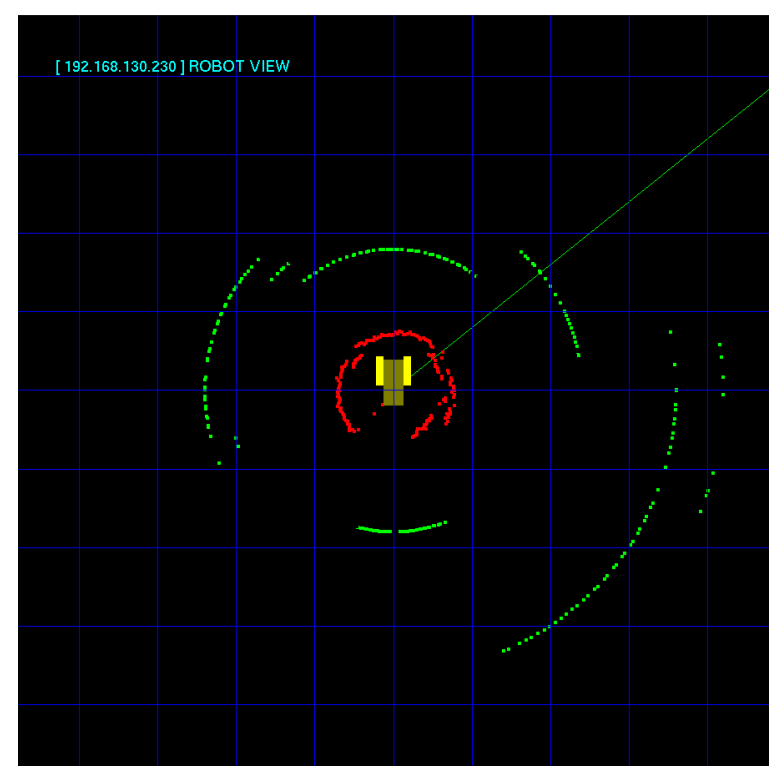

Fig. 10. CMFA-filtered UWB radar (green) and LIDAR (red) readings in completely fogged environment

We then tested simple collision avoidance behaviors using both radar and LIDAR. With either sensor, the robot moves forward until the range to the closest obstacle ahead drops below a specified threshold. Both behaviors worked flawlessly in clear air, driving to the specified distance from the forward wall and stopping. In a fog-filled environment, the LIDAR was unable to see through the fog, so the robot was unable to move. In contrast, the UWB radar was able to see through the fog, so the robot was able to drive to the specified distance from the wall and stop. There was no difference in the performance of the radar-based collision avoidance behavior in clear air and in dense fog.

\section{CONCLUSIONS AND FUtURE WORK}

Our experiments show that UWB radar can provide useful perception capabilities for small, man-portable UGVs under all-weather conditions. Unlike optical sensors, such as LIDAR and vision, UWB radar can penetrate dense fog to detect obstacles. Unlike conventional radars (such as automotive radars), UWB radar provides precise range information, allowing perception systems to determine the size and shape of detected obstacles. In addition, UWB radar is effective at sensing objects that are stationary with respect to the sensor, unlike many types of radar that can only detect moving obstacles. Finally, due to the low power and precise ranging of UWB radars, they are effective for use indoors as well as outdoors and can allow robots to avoid collisions in cluttered environments.

Future work will include improving the accuracy of UWB radar perception algorithms, fusing UWB radar data with LIDAR and stereo vision data, and integrating all of these perception capabilities within an autonomous navigation system. We will investigate the use of occupancy grids to allow the robot to build maps over time that increase the effective angular resolution of the radar sensor and fuse radar data with LIDAR and stereo vision data. We will also implement integrated capabilities for waypoint navigation, path planning, and autonomous exploration for the Daredevil navigation system.

\section{ACKNOWLEDGMENTS}

We thank Erik Schoenfeld for performing the hardware integration for the Daredevil PackBot and Jon Brookshire for writing the Linux-based RaDeKL UWB driver software.

\section{REFERENCES}

[1] K. Konolige, "ERRATIC competes with the big boys," AI Magazine, vol. 16, no. 2, pp. 61-67, Summer 1995.

[2] L. Matthies, Y. Xiong, R. Hogg, D. Zhu, A. Rankin, B. Kennedy, M. Hebert, R. Maclachlan, C. Won, T. Frost, G. Sukhatme, M. McHenry, and S. Goldberg, "A portable, autonomous, urban reconnaissance robot," Robotics and Autonomous Systems, vol. 40, pp. 163-172.

[3] E. Pacis, H.R. Everett, N. Farrington, D. Bruemmer, "Enhancing functionality and autonomy in man-portable robots," in Proc. SPIE, vol. 5422, April 2004, pp. 355-366.

[4] B. Yamauchi, "Wayfarer: An autonomous navigation payload for the PackBot," Proc. AUVSI Unmanned Vehicles North America, June 2005.

[5] M. Schneider, "Automotive radar - Status and trends," in Proc. German Microwave Conference (GeMic 2005), Ulm, Germany, 2005.

[6] B. Yamauchi, 'Daredevil: Ultra wideband radar sensing for small UGVs," in Proc. SPIE, vol. 6561, April 2007, pp. 65610B1656100B9.

[7] R.J. Fontana, "Recent systems applications of short-pulse ultrawideband (UWB) technology," IEEE Trans. Microwave Theory and Techniques, vol. 52, no. 9, pp. 2087-2104, September 2004.

[8] C. Urmson, et al., "Autonomous driving in urban environments: Boss and the Urban Challenge," Journal of Field Robotics, 25(8), pp. 425 466, August 2008.

[9] M. Montemerlo, et al., "Junior: The Stanford entry in the Urban Challenge," Journal of Field Robotics, 25(9), pp. 569-597, September 2008

[10] J. Leonard, et al., "A perception-driven autonomous urban vehicle," Journal of Field Robotics, 25(10), pp. 727-774, October 2008.

[11] T. Strobel and A. Servel. (2004, July 19). Sensor data sheets - Stateof-the-art of sensors and sensor data fusion for automotive preventive safety applications [online]. Available: http://www.prevent-ip.org/download/deliverables/ProFusion/PR13400-IPD-040531-v10-Sensor Data Sheets.pdf

[12] H. Moravec and A. Elfes, "High resolution maps from wide angle sonar." In Proc. IEEE International Conference on Robotics and Automation, March 1985, pp. 116-121. 